\title{
Biomechanical Comparison of Meniscal Allograft Root Fixation Techniques: Anterograde Interference Bone Plug Fixation Yields Favorable Results Compared to Transosseous Suture Fixation Alone
}

\author{
Andrzej Brzezinski, M.D, Casey Imbergamo, M.D, William Pfaff, Ph.D., Rae Tarapore, B.A., \\ Matthew Nasra, B.A., Michael Simon, B.A., and Charles Gatt, M.D.
}

\begin{abstract}
Purpose: To compare the biomechanical properties of 2 different fixation techniques (interference bone plug fixation vs transosseous suture fixation) of the posterior horn of the medial meniscus using a porcine model. Methods: Twenty-six matched pairs of fresh-frozen juvenile domestic porcine knees were used in this study. Specimens were randomly distributed among 3 groups: (1) native meniscus groups, (2) interference fixation, and (3) transosseous suture fixation. In each group, the posterior segments of the tested medial menisci were gripped with the freeze clamps and fixed to the tensile testing machine. Samples were preconditioned, followed by cyclic tension-relaxation for 1000 cycles between 10 and $30 \mathrm{~N}$ at $0.5 \mathrm{~Hz}$ and finally pulled to failure at a rate of $0.55 \mathrm{~mm} / \mathrm{s}$. The cyclic elongation, stiffness to failure, mode, and ultimate load to failure were recorded. Results: There was no significant difference in ultimate load to failure between the interference fixation $(169.71 \pm 71.98 \mathrm{~N})$ and transosseous suture fixation $(222.73 \pm 72.40 \mathrm{~N})$ groups $(P=.118)$, both were significantly less than that of the native meniscus $(405.46 \pm 95.62)(P<.001)$. Interference fixation displayed cyclic elongation $(1.04 \pm 0.71 \mathrm{~mm})$ and stiffness $(69.10 \pm 25.8 \mathrm{~N} / \mathrm{mm})$ that were not significantly different from the native meniscus tissue $(0.78 \pm 0.53 \mathrm{~mm}$ and $83.1 \pm 26.28 \mathrm{~N} / \mathrm{mm})(P=.359$ and $P=.224)$, in comparison to transosseous suture fixation, which did show increased cyclic elongation $(1.85 \pm 1.44 \mathrm{~mm})(P=.047)$ and decreased stiffness $(34.72 \pm 10.2$ $\mathrm{N} / \mathrm{mm})(P<.001)$. Conclusion: Interference fixation of the posterior horn of the medial meniscus has superior cyclic elongation and stiffness when compared to transosseous suture fixation. Interference fixation and the native meniscus model have a similar stiffness and cyclic elongation. Clinical Relevance: The significance of our study is that using interference fixation for meniscal allograft transplantation has the potential to reduce short term surgical failures as well as long term complication rates.
\end{abstract}

$\mathbf{T}$ he meniscus is a load-bearing tissue that plays an important role in intra-articular force distribution

\footnotetext{
From the Rutgers-Robert Wood Johnson Medical School (A.B., R.T., M.N., M.S.C.G.), New Brunswick, New Jersey; and MedStar Union Memorial Hospital (C.I.), Baltimore, Maryland, U.S.A.

The authors report that they have no conflicts of interest in the authorship and publication of this article. Full ICMJE author disclosure forms are available for this article online, as supplementary material.

This project was funded by the Rutgers Robert Wood Johnson Medical School Department of Orthopaedic Surgery.

Received October 6, 2021; accepted January 3, 2022.

Address correspondence to Rae Tarapore, B.A., 125 Paterson St New Brunwsick, NJ,08901.E-mail: raetarapore@gmail.com

(C) 2022 THE AUTHORS. Published by Elsevier Inc. on behalf of the Arthroscopy Association of North America. This is an open access article under the CC BY-NC-ND license (http://creativecommons.org/licenses/by-nc-nd/4.0/). 2666-061X/211370

https://doi.org/10.1016/j.asmr.2022.01.001
}

and stabilization of the knee. The intact meniscus allows for transformation of the axially oriented compression forces generated across the knee into hoop stresses within the meniscal tissue that dissipate onto the tibial plateau via the meniscal roots. Fifty percent of weightbearing on the medial side of the knee is transmitted through the medial meniscus. ${ }^{1}$ Meniscal injuries are common, and over half a million meniscectomies are performed yearly in the United States. ${ }^{2}$ Meniscectomies were found to be successful for the short-term improvement of pain in patients with nonreparable meniscal tears; however, they are linked to long-term complications such as progression of osteoarthritis. ${ }^{3-5}$ Meniscal allograft transplantation (MAT) has become a viable treatment option for symptomatic patients with post-meniscectomy syndrome. The first MAT was performed in 1984, and since then various surgical techniques have been developed including arthroscopic- 
assisted methods. ${ }^{6}$ The role of MAT is to restore knee biomechanics and prevent or delay osteoarthritis. ${ }^{7}$ To restore chondroprotective function the meniscal allograft needs to be adequately sized and attached to the tibial plateau. ${ }^{8}$ There are various fixation techniques for medial MAT. The most common technique used is individually fixing the anterior and posterior meniscal roots to the tibia via transosseous sutures. Some surgeons retain bone plugs with the meniscal roots, and some implant the allograft without bone plug. ${ }^{9}$ Some studies have found that retention of the bone plugs for fixation of medial MAT yielded superior results compared to implantation of an MAT without bone plugs in terms of improved tibiofemoral contact pressures. ${ }^{10,11}$ However, no definitive gold standard technique has been established to date. ${ }^{12}$ In our laboratory we are developing a technique for interference fixation of the posterior horn of a meniscus transplant. In this technique, the posterior root and bone plug are fixed into its tunnel with an interference screw inserted over a guidewire placed through the posteromedial portal. The anterior horn will also be fixed with interference screw fixation, but this will be performed with direct visualization through the mini arthrotomy that is used to insert the meniscal allograft. The primary mode of failure of MAT is extrusion of the implant. The extrusion of the allograft results in increased joint contact forces $^{13,14}$ and is believed to be the result of less-thanoptimal root fixation. ${ }^{15,16}$ The identification of an ideal tibial fixation technique has the potential to help optimize MAT procedures and reduce failure rates.

We compared interference fixation (IF) of the bone plug to transosseous suture fixation (TSF) of the posterior horn of the medial meniscus using a porcine model. To identify any potential differences between the two fixation constructs, this study examined cyclic elongation, stiffness, and ultimate tensile load to failure. The ideal fixation construct would exhibit minimal cyclic elongation to decrease risk of meniscal allograft extrusion, maximal stiffness to allow for a greater proportion of tibiofemoral load to be transferred to the meniscus, and a high ultimate tensile load to failure. The purpose of this study was to compare the biomechanical properties of 2 different fixation techniques (interference bone plug fixation vs transosseous suture fixation) of the posterior horn of the medial meniscus using a porcine model. The null hypothesis was that there would be no significant difference between IF and TSF fixation constructs in response to dynamic and ultimate tensile stresses.

\section{Methods}

\section{Specimen Preparation}

Twenty-six matched pairs of fresh-frozen juvenile (within 6 to 10 months of age) domestic porcine knees were used in this study. Each was obtained from a local slaughterhouse. Porcine knee models were chosen because they have been used extensively in previous biomechanical testing studies for meniscus fixation. Generally, porcine knees have bone density closer to that of young human subjects who are candidates for MAT. Additionally, they are also more readily available and cost-effective in comparison to human cadaveric knees. 2,3,5,17-19 All knees were inspected for signs of meniscal damage, cartilage deterioration, and other defects before testing. Each knee was disarticulated, and tibias were isolated for further dissection. All soft tissue was removed from the bone except the medial meniscus, which was left intact on the tibial plateau. The anterior half of the medial meniscus was resected, and the posterior segment with its bony attachment was preserved. Each specimen was potted in a cylindrical mold using poly-methyl methacrylate cement (Fricke Dental International Inc., Streamwood, IL) to a point $4 \mathrm{~cm}$ distal to the proximal aspect of the tibial tuberosity. 7,20

\section{Testing model}

Specimens were randomly distributed among 3 groups: (1) native meniscus, (2) IF, and (3) TSF groups, with 12 knees for native meniscus group and 20 for each fixation construct. The medial menisci segments were left intact for specimens assigned to the native tissue group. In both fixation groups, half of the specimens were used as meniscal donors and half as testing specimens. A $2 \times 2 \mathrm{~cm}$ bone block of the tibial plateau with attached medial meniscus was harvested from each of the donor's knees. The bone block was then shaped into a $10 \mathrm{~mm}$ long bone plug of diameter equal to the meniscal root width that was on average $9.4 \mathrm{~mm}$ (9-10) (Fig 1). Finally, each of the harvested menisci was randomly assigned for implantation into the remaining tibias. Tibial fixation was performed for both experimental groups by the same orthopedic surgeon. Using an Acufex Anatomic ACL Guide System (Smith \& Nephew, London, UK), a transtibial tunnel was created in each testing specimen. Tunnels were drilled from the anteromedial cortex of the tibial metaphysis through the footprint of the posterior horn of the meniscus on the tibial plateau. ${ }^{3,15,21}$ The inclination of the tunnel was $55^{\circ}$, and the diameter was either $9 \mathrm{~mm}$ or $10 \mathrm{~mm}$ based on the measured diameter of the bone plug. The average tunnel length was $37.7 \mathrm{~mm}(35-47 \mathrm{~mm})$. A braided polyester nonabsorbable suture (No. 5 Ethibond; Ethicon, Bridgewater, NJ) was passed through the bone plug and fed through the transtibial tunnel to pull the bone plug into place and aid with fixation. To secure the meniscus in the IF group a titanium suture anchor (with sutures removed) (TwinFix Ultra Ti $5.5 \mathrm{~mm}$ Suture Anchor; Smith \& Nephew) was placed in the proximal end of the tunnel between the bone plug 


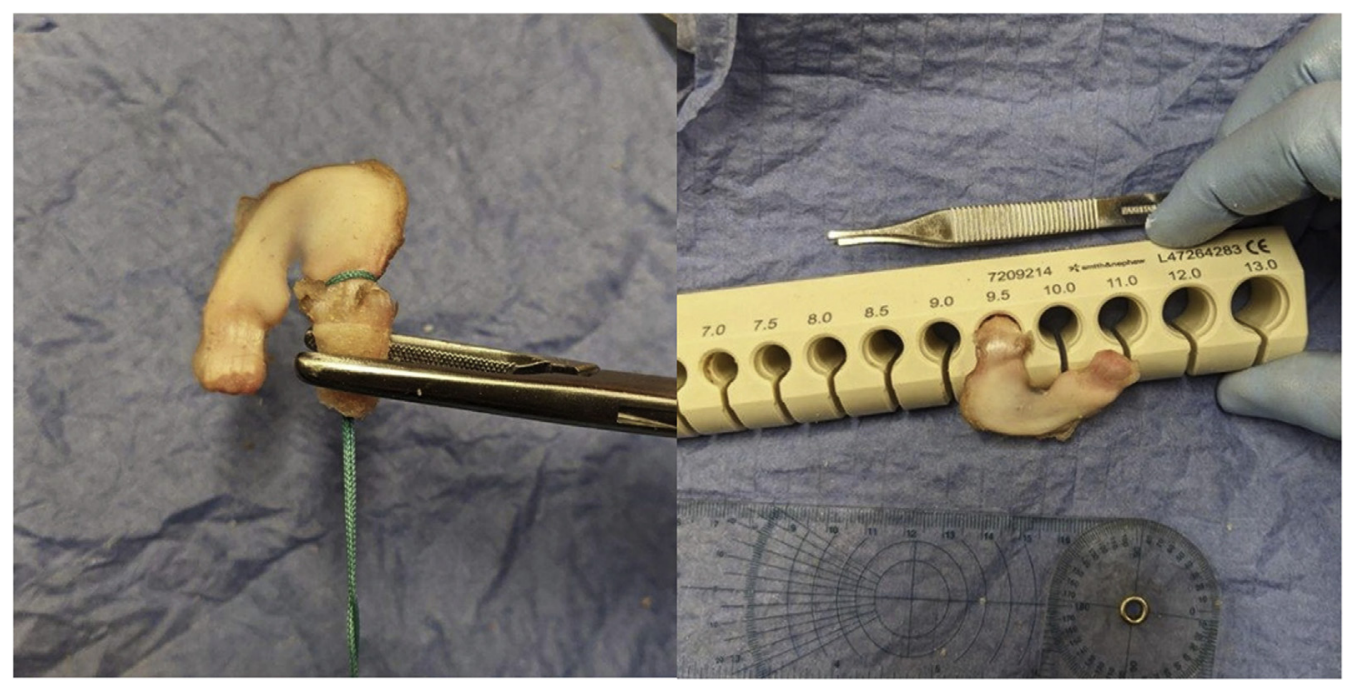

Fig 1. Medial meniscal allograft with $9.5 \mathrm{~mm}$ bone plug.

and the tunnel interface in an anterograde fashion. ${ }^{5}$ The titanium anchor was selected for interference fixation because its shape is similar to that of an interference screw, and our laboratory has been successful inserting the anchor through a posteromedial portal into the medial meniscal root attachment site in a human cadaveric model. For the TSF group the strands of suture were firmly manually tensioned and tied over an extracortical button (Endobutton; Smith $\&$ Nephew) at the distal end of the tunnel with 10 throws to ensure that the knots were not a source of failure.

\section{Biomechanical Testing}

Each knee was secured to a custom fixture and clamped to the base of a dynamic tensile testing machine (5564 Instron Material Test Machine; Lawrence Instron Corporation, Norwood, MA). The fixture allowed for positioning of the tibia to be adjusted so that the force vector was in line with the transtibial tunnel and perpendicular to the base [1]. The medial meniscus was then clamped and rigidly fixed to the actuator of the tensile testing machine using freeze clamps (Electroforce; TA Instruments, New Castle, DE) (Fig 2). All samples were hydrated before testing and during the testing process using gauze pads soaked in phosphatebuffered saline solution. All 3 groups were subject to the same cyclic tension-relaxation protocol: specimens were preconditioned for 10 cycles between 1 and $10 \mathrm{~N}$ at $0.1 \mathrm{~Hz}$, followed by cyclic tension-relaxation for 1000 cycles between 10 and $30 \mathrm{~N}$ at $0.5 \mathrm{~Hz}^{1,7,8,15,21}$ This loading protocol has been used by previous studies to approximate the tensile forces on the posterior medial meniscus root under neutral rotation, range of motion from $0^{\circ}$ to $90^{\circ}$ of knee flexion, and $500 \mathrm{~N}$ of tibiofemoral load, which we believe to be representative of range of motion and partial weightbearing seen in postoperative rehabilitation regimens after meniscal allograft transplantation. ${ }^{7,8,21,22}$ Displacement of the meniscus was recorded at the actuator of the tensile testing machine at the conclusion of cycles 1, 100, 500, and 1000, similar to previous studies evaluating displacement in meniscus tissue. ${ }^{7,8}$ On completion of the cyclic loading protocol, the menisci were pulled to failure at a rate of $0.5 \mathrm{~mm} / \mathrm{s}^{22}$ Mode of failure was recorded as suture breakage, bone block failure, bone block slippage, or meniscal tissue failure.

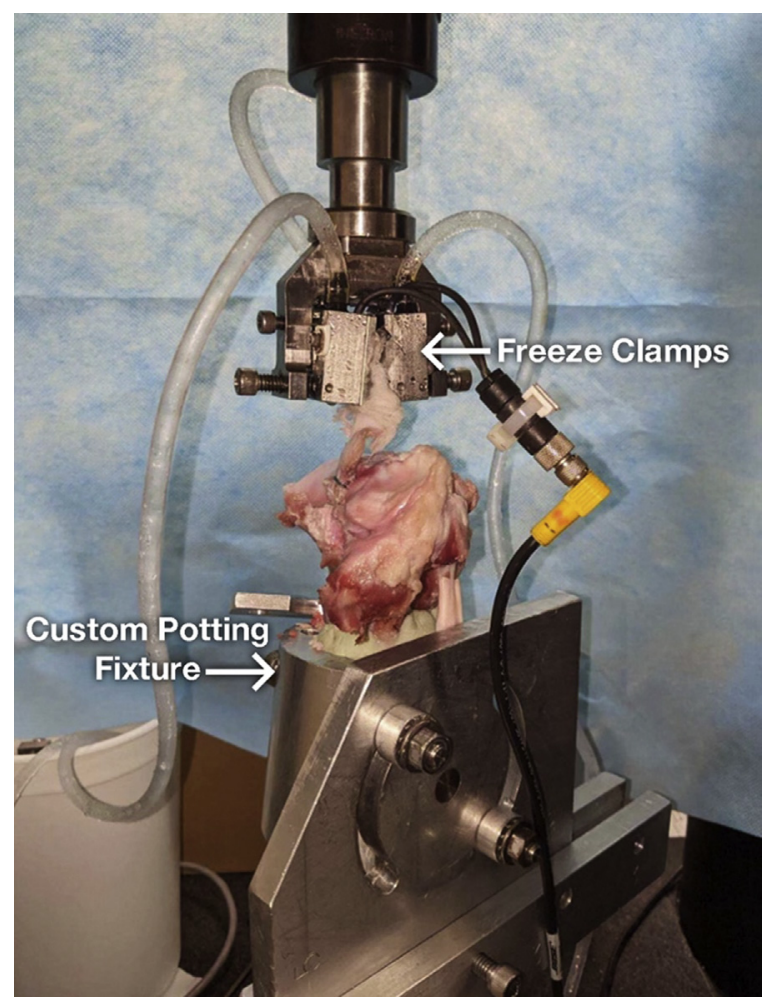

Fig 2. Porcine knee secured in a custom potting fixture with the meniscus grasped by freeze clamps for dynamic tensile testing. 


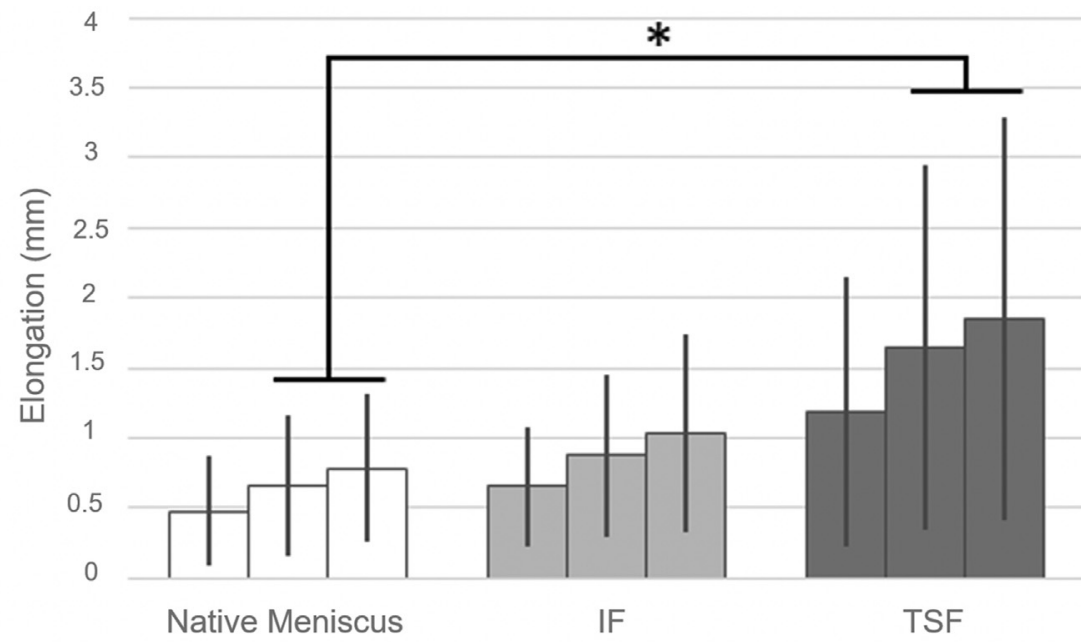

Fig 3. Cyclic elongation at 100,500 , and 1000 cycles. Asterisk denotes significant difference between groups.

\footnotetext{
* Denotes significant difference between groups
}

\section{Calculations and Statistical Analysis}

Cyclic elongation was calculated as the difference in extension between the end of the first cycle and the end of the 1000th cycle. From the ultimate tensile test, stiffness was calculated from the slope of the load deformation curve in the elastic region of the curve. All parameters were compared between the two fixation experimental groups and the native tissue control group using l-way analysis of variance followed by paired $t$-tests to determine statistical significance. Twotail $P$ values $<.05$ and $<.001$ were considered significant and highly significant, respectively. All analyses were performed using Stata 16 software (StataCorp, College Station, TX) and Microsoft Excel (Microsoft, Redmond, WA).

\section{Results}

\section{Cyclic Elongation}

There was a significant difference in cyclic elongation between the TSF and native meniscus groups, with the TSF group displaying $137 \%$ greater elongation $(P=$ .047). The average cyclic elongation of the TSF group was $1.85 \pm 1.44 \mathrm{~mm}$ compared to $0.78 \pm 0.53 \mathrm{~mm}$ for the native meniscus group. There was no significant difference between the IF group and both the native meniscus $(P=.359)$ and TSF groups $(P=.132)$ (Fig 3$)$.

\section{Stiffness}

There was a significant difference in stiffness between the TSF group and the IF $(P=.002)$, and between the TSF and native meniscus groups $(P<.001)$. The IF group exhibited stiffness that was $83.2 \%$ of the native meniscus group, and the TSF group exhibited stiffness that was $41.8 \%$ of the native meniscus group. There was no significant difference between the IF and native meniscus groups $(P=.224)$ (Fig 4$)$.

\section{Ultimate Load to Failure}

There was a significant difference in ultimate load to failure between the native meniscus group and both the IF $(P<.001)$ and TSF groups $(P<.001)$. The interference fixation group exhibited failure at a level that was $41.9 \%$ of the native meniscus group, and the TSF group exhibited failure at a level that was $54.9 \%$ of the native meniscus group. There was no significant difference between the interference fixation and TSF groups $(P=.118)$ (Table 1$)$.

\section{Mode of Failure}

There was a difference in the mode of failure between the testing groups. For the native meniscus, all specimens failed by tearing at the root. All specimens in the TSF fixation group experienced a break of the polyester suture over the cortical button. Interference fixation failure occurred from breakage and slippage of the bone plug (Fig 5). Four specimens failed from slippage of the bone plug, 2 specimens failed from isolated breakage of the bone plug, and 4 failed from simultaneous breakage and slippage of the bone plug.

\section{Discussion}

Our study found that the interference fixation of the posterior horn of the meniscus had superior stiffness and cyclic elongation when compared to transosseous suture fixation. There was no difference in ultimate load between the 2 groups, both of which had an ultimate load that was significantly less than that of the native meniscus group. 
Fig 4. Stiffness by group. Asterisk denotes significant difference between groups.

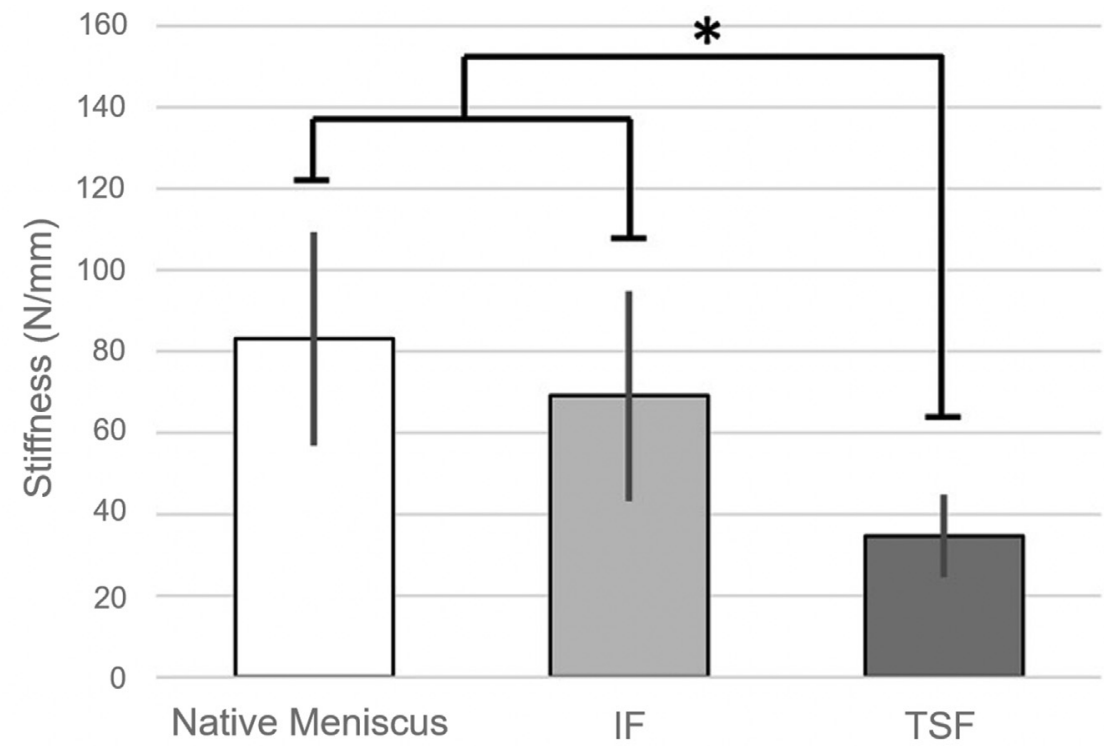

* Denotes significant difference between groups
Meniscal allograft transplantation (MAT) has become a potential treatment option for patients with postmeniscectomy syndrome over the past few years, which is broadly characterized by intractable pain after partial or complete meniscectomy. ${ }^{23}$ Because of the wide variety of techniques and materials considered, standardizing an approach to performing this procedure may potentially reduce high complication rates while optimizing clinical outcomes. ${ }^{12}$ This study aimed to examine the biomechanical properties of meniscal allograft bone plug interference and transosseous suture fixation of the posterior medial meniscal horn as a means of determining a superior method for MAT tibial fixation. TSF was chosen because it is a commonly used surgical technique, and there are several preclinical studies evaluating this technique. ${ }^{5}$ IF was chosen because this technique has been evaluated for a tissue engineered meniscus replacement. ${ }^{5}$ Additionally, our laboratory has completed pilot work demonstrating the feasibility of arthroscopically assisted antegrade fixation of the posterior horn of a meniscal allograft through a posteromedial portal. Our results showed that IF had superior biomechanical properties in comparison to TSF. Specifically, IF displayed cyclic elongation and stiffness that were comparable to native meniscus tissue.

Increased elongation during cyclic loading can result in meniscal allograft extrusion, which in turn will decrease meniscal hoop tension and lead to increased femorotibial contact stress. ${ }^{19,24}$ As a result, the ideal tibial fixation method should demonstrate minimal cyclic elongation that maintains the biomechanical integrity of the allograft and surrounding tissue. Our study showed that IF exhibited less elongation than TSF, with only a $33.3 \%$ increase compared to native meniscus tissue, which was not significantly different. Although the difference in elongation between IF and TSF was small $(0.8 \mathrm{~mm})$, this may have a significant impact on meniscal extrusion. Greater than $3 \mathrm{~mm}$ of meniscal extrusion is associated with inferior clinical outcomes in MAT. ${ }^{25,26}$ Thus we believe that the difference in cyclic elongation between IF and TSF are clinically, as well as statistically significant. Our findings are consistent with those reported by Patel et al. ${ }^{5}$ in a meniscal device tibial fixation study, which found that posterior medial screw fixation displayed elongation only $36.5 \%$ greater than native meniscus roots. Previous studies have compared interference screw to suture fixation in tibial fixation of ACL grafts, and while these studies examined a different type of graft, they similarly found that the interference screw exhibited significantly less cyclic elongation than suture fixation. ${ }^{27,28}$

The favorable elongation properties of the IF may be attributed to the stiffness of the fixation construct. ${ }^{5}$ The interference fit technique used in our study exhibited stiffness that was comparable to the native meniscus, likely caused by the frictional interference created by

Table 1. Ultimate Load to Failure by Group

\begin{tabular}{lccc}
\hline \multicolumn{1}{c}{ Group } & Count & Average $(\mathrm{N})$ & Standard Deviation $(\mathrm{N})$ \\
\hline Native meniscus & 12 & 405.46 & 95.62 \\
IF & 10 & 169.71 & 71.98 \\
TSF & 10 & 222.73 & 72.40 \\
\hline
\end{tabular}




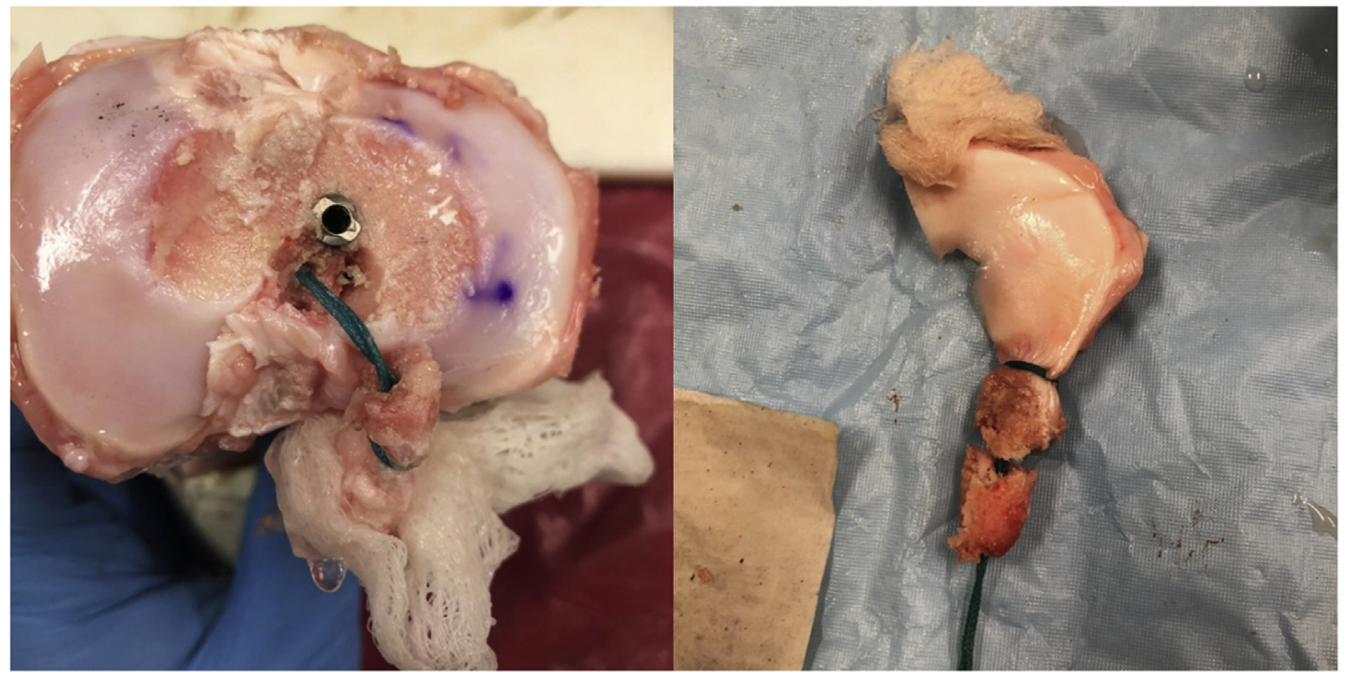

Fig 5. Bone plug breakage as the mode of failure with interference fixation. the screw. Alternatively, the suture used in the TSF group was presumably the limiting factor, leading to decreased stiffness (58.2\% less than native meniscus) and subsequent increased elongation. A previous study by Patel at al. ${ }^{5}$ comparing interference screw to transosseous suture fixation of a synthetic meniscal replacement device yielded similar results, with the interference screw exhibiting greater stiffness. The combination of increased stiffness and decreased elongation displayed by the interference screw would provide superior tibiofemoral contact stress mechanics and limit extrusion of meniscus allograft.

Although there was no significant difference in ultimate load to failure between the IF and TSF groups, both were approximately half of the native meniscus values. The decreased load to failure in the TSF group can be attributed to suture breakage. In the IF group, most failures occurred because of bone plug breakage and subsequent slippage from the bony tunnel, which may be related to the technique implantation. Although we did not demonstrate a difference between IF and TSF, the load to failure occurred above the load generated during the early rehabilitation phase of the MAT. Because this is a cadaveric, time zero study, it does not account for the biologic ingrowth of the allograft bone plug. At later time points in the in vivo model, the mechanical fixation supplied by the interference device or the transosseous sutures should be irrelevant.

\section{Limitations}

This present study was not without limitations. First, we were unable to consider the density of the bones that we used during this study. This parameter may be influential in interference mechanics. In clinical practice, bone density may need to be considered because of its potential mechanical significance. Furthermore, this study used porcine knee models as opposed to human cadaveric knee models. The results of this study may not be directly generalizable to human tissue, but they do serve as a preliminary baseline upon which further research can be performed. Additionally, this study only used 1 type of braided polyester suture, and it is possible that exploring various suture types may have yielded different results for the TSF group. However, it has been previously documented that similar material properties exist between different types and sizes of sutures in orthopaedics. ${ }^{29}$ Also, the interference screw used for the testing was slightly undersized compared to the current recommendations for use in clinical practice, which might affect the ultimate load to failure in the IF group. ${ }^{30}$ Increasing the diameter of the interference device might increase the incidence of posterior tunnel blowout. In this study we did use a titanium suture anchor since we had performed pilot work demonstrating the antegrade insertion feasibility from a posteromedial portal. Later pilot work has demonstrated the feasibility of antegrade insertion of a cannulated titanium interference screw over a flexible guide wire, again from a posteromedial portal. Last, there was variability in the porcine knee models regarding age and gender that led to a subsequent variability in tested meniscal tissue. The current findings show that IF of the posterior horn of the medial meniscus yielded favorable results and should be considered in future studies exploring the implantation of meniscal allografts. 


\section{Conclusions}

Interference fixation of the posterior horn of the medial meniscus has superior cyclic elongation and stiffness when compared to transosseous suture fixation. Interference fixation and the native meniscus model have a similar stiffness and cyclic elongation.

\section{Acknowledgments}

The authors thank Jorden Xavier, Jason Yang, and Barbara Perry.

\section{References}

1. Fox AJ, Bedi A, Rodeo SA. The basic science of human knee menisci: structure, composition, and function. Sports Health 2012;4:340-351.

2. Sihvonen R, Paavola M, Malmivaara A, et al. Arthroscopic partial meniscectomy for a degenerative meniscus tear: A 5 year follow-up of the placebo-surgery controlled FIDELITY (Finnish Degenerative Meniscus Lesion Study) trial. Br J Sports Med 2020;54:1332-1339.

3. Allen PR, Denham RA, Swan AV. Late degenerative changes after meniscectomy. Factors affecting the knee after operation. J Bone Joint Surg Br 1984;66:666-671.

4. Wilson W, van Rietbergen B, van Donkelaar CC, Huiskes R. Pathways of load-induced cartilage damage causing cartilage degeneration in the knee after meniscectomy. J Biomech 2003;36:845-851.

5. Patel JM, Brzezinski A, Raole DA, Dunn MG, Gatt CJ Jr. Interference screw versus suture endobutton fixation of a fiber-reinforced meniscus replacement device in a human cadaveric knee model. Am J Sports Med 2018;46: 2133-2141.

6. Zhang YD, Hou SX, Zhong HB, Zhang YC, Luo DZ. Meniscal allograft transplantation using a novel allarthroscopic technique with specifically designed instrumentation. Exp Ther Med 2018;15:3020-3027.

7. Doral MN, Bilge O, Huri G, Turhan E, Verdonk R. Modern treatment of meniscal tears. EFORT Open Rev 2018;3: 260-268.

8. Seitz AM, Dürselen L. Biomechanical considerations are crucial for the success of tendon and meniscus allograft integration-A systematic review. Knee Surg Sports Traumatol Arthrosc 2019;27:1708-1716.

9. Figueroa F, Figueroa D, Calvo R, Vaisman A, EspregueiraMendes J. Meniscus allograft transplantation: Indications, techniques and outcomes. EFORT Open Rev 2019;4: 115-120.

10. Ambra LF, Mestriner AB, Ackermann J, Phan AT, Farr J, Gomoll AH. Bone-plug versus soft tissue fixation of medial meniscal allograft transplants: A biomechanical study. Am J Sports Med 2019;47:2960-2965.

11. Rodeo SA. Meniscal allografts-Where do we stand? Am J Sports Med 2001;29:246-261.
12. Gilat R, Cole BJ. Meniscal allograft transplantation: Indications, techniques, outcomes. Arthroscopy 2020;36: 938-939.

13. Ode GE, Van Thiel GS, McArthur SA, et al. Effects of serial sectioning and repair of radial tears in the lateral meniscus. Am J Sports Med 2012;40:1863-1870.

14. Rao AJ, Erickson BJ, Cvetanovich GL, Yanke AB, Bach BR Jr, Cole BJ. The meniscus-deficient knee: Biomechanics, evaluation, and treatment options. Orthop J Sports Med 2015;3:2325967115611386.

15. LaPrade CM, Foad A, Smith SD, et al. Biomechanical consequences of a nonanatomic posterior medial meniscal root repair. Am J Sports Med 2015;43:912-920.

16. Abat F, Gelber PE, Erquicia JI, Pelfort X, GonzalezLucena G, Monllau JC. Suture-only fixation technique leads to a higher degree of extrusion than bony fixation in meniscal allograft transplantation. Am J Sports Med 2012;40:1591-1596.

17. Feucht MJ, Grande E, Brunhuber J, Burgkart R, Imhoff AB, Braun S. Biomechanical evaluation of different suture techniques for arthroscopic transtibial pull-out repair of posterior medial meniscus root tears. Am J Sports Med 2013;41:2784-2790.

18. Feucht MJ, Grande E, Brunhuber J, et al. Biomechanical evaluation of different suture materials for arthroscopic transtibial pull-out repair of posterior meniscus root tears. Knee Surg Sports Traumatol Arthrosc 2015;23: 132-139.

19. Feucht MJ, Grande E, Brunhuber J, et al. Biomechanical comparison between suture anchor and transtibial pullout repair for posterior medial meniscus root tears. Am J Sports Med 2014;42:187-193.

20. Lee SJ, Aadalen KJ, Malaviya P, et al. Tibiofemoral contact mechanics after serial medial meniscectomies in the human cadaveric knee. Am J Sports Med 2006;34: 1334-1344.

21. LaPrade CM, LaPrade MD, Turnbull TL, Wijdicks CA, LaPrade RF. Biomechanical evaluation of the transtibial pull-out technique for posterior medial meniscal root repairs using 1 and 2 transtibial bone tunnels. Am J Sports Med 2015;43:899-909.

22. LaPrade RF, LaPrade CM, Ellman MB, Turnbull TL, Cerminara AJ, Wijdicks CA. Cyclic displacement after meniscal root repair fixation: a human biomechanical evaluation. Am J Sports Med 2015;43:892-898.

23. Drobnič M, Ercin E, Gamelas J, et al. Treatment options for the symptomatic post-meniscectomy knee. Knee Surg Sports Traumatol Arthrosc 2019;27:1817-1824.

24. Stärke C, Kopf S, Gröbel KH, Becker R. The effect of a nonanatomic repair of the meniscal horn attachment on meniscal tension: A biomechanical study. Arthroscopy 2010;26:358-365

25. Ha JK, Shim JC, Kim DW, Lee YS, Ra HJ, Kim JG. Relationship between meniscal extrusion and various clinical findings after meniscus allograft transplantation. Am J Sports Med 2010;38:2448-2455.

26. Lee SM, Bin SI, Kim JM, et al. Long-term outcomes of meniscal allograft transplantation with and without 
extrusion: Mean 12.3-year follow-up study. Am J Sports Med 2019;47:815-821.

27. Scheffler SU, Südkamp NP, Göckenjan A, Hoffmann RF, Weiler A. Biomechanical comparison of hamstring and patellar tendon graft anterior cruciate ligament reconstruction techniques: The impact of fixation level and fixation method under cyclic loading. Arthroscopy 2002;18: 304-315.

28. Tetsumura S, Fujita A, Nakajima M, Abe M. Biomechanical comparison of different fixation methods on the tibial side in anterior cruciate ligament reconstruction: A biomechanical study in porcine tibial bone. J Orthop Sci 2006;11:278-282.

29. Najibi S, Banglmeier R, Matta J, Tannast M. Material properties of common suture materials in orthopaedic surgery. Iowa Orthop J 2010;30:84-88.

30. Butler JC, Branch TP, Hutton WC. Optimal graft fixation-The effect of gap size and screw size on bone plug fixation in ACL reconstruction. Arthroscopy 1994;10: 524-529. 